\title{
Hydrogel keratophakia: a microkeratome dissection in the monkey model
}

\author{
W HOUDIJN BEEKHUIS, ${ }^{2}$ BERNARD E McCAREY,${ }^{\circ}$ GEORGE O WARING, \\ AND GABRIEL VAN RIJ ${ }^{2}$ \\ From the 'Department of Ophthalmology, Emory University, Atlanta, Georgia 30322, USA, and ${ }^{2}$ Erasmus \\ University, Rotterdam, The Netherlands
}

SUMMARY High water content intracorneal implants were fabricated from Vistamarc hydrogel (Vistakon, Inc.) at $58 \%, 68 \%$, and $72 \%$ water content and a range of powers from $+7 \cdot 25$ to $+17 \cdot 00$ dioptres. The Barraquer microkeratome technique was used to implant the lens at $59.0 \pm 9 \%$ ( \pm SD) depth in the corneas of 14 rhesus monkey eyes. The contralateral eye served as a control. Three eyes were lost to the study because of complications. The remaining 11 animals were followed up for $51 \pm 2$ weeks with the refractive yield being $118 \pm 34 \%$ and the keratometric yield being $92 \pm 30 \%$. The measured and theoretically expected refractive changes have a linear regression line correlation coefficient of $0 \cdot 74$, whereas the respective keratometric data had a correlation coefficient of 0:04. The measured refraction became stable within 2 to 3 dioptres after 20 postoperative weeks. The hydrogels were well tolerated within the corneal tissue. There was a minimum of interface problems except along the edge of the implant. Implants with abruptly cut edges versus a fine wedge tended to have more light scattering collagen at the implant margin.

Refractive keratoplasty with hydrogel implants has the potential to alter the anterior corneal curvature in the correction of either hypermetropia or myopia. The implants could be manufactured in limitless supply with well controlled parameters. Implanting high water content hydrogel lenses via a free-hand lamellar pocket dissection changes the refraction in rabbits $^{1}$ but not significantly in primate corneas. ${ }^{2}$ Barraquer $^{3}$ proposed to use the microkeratome to produce a lamellar dissection that would cut the relatively non-elastic collagen bundles and Bowman's layer. The lamellar disc can be contoured over the intrastromal lens, thus altering the anterior corneal surface curvature. Binder et al. ${ }^{4}$ found that hydrogel plus-power lenses implanted in a microkeratome incision could cause significant corneal topography steepening.

This paper reports the detailed results of hydrogel keratophakia by Barraquer's microkeratome technique in rhesus monkeys with lenses of three different water contents and various plus powers. The mean follow-up time of $\mathbf{1 2}$ months allowed evalu-

Correspondence to Bernard E McCarey, PhD, Emory University Eye Center, Room No. 3615 South, 1327 Clifton Road, NE, Atlanta, Ga. 30322, USA. ation of the predictability of the procedure as well as correlation of the keratometric and refractive changes relative to the parameters of the hydrogel lenticule.

\section{Materials and methods}

INTRACORNEAL LENS PARAMETERS

The parameters of the intracorneal lenses (ICL) that were implanted are listed in Table 1 . A total of 14 eyes received Vistamarc (Vistacon) lenses with water contents of $58 \%$ (six eyes), $68 \%$ (three eyes) and $72 \%$ (five eyes). The power $(7.25$ to $+17.00 \mathrm{D})$, base curve $(6.50$ to $7.2 \mathrm{~mm})$, and diameter ( 4.5 to $5.8 \mathrm{~mm})$ of these lenses as indicated by the manufacturer were used in determining the theoretical refractions and keratometer values. ${ }^{2}$ The manufacturer measured the hydrated lens power with a lensometer after blotting the surface water from the lens. It is difficult to measure the power of the hydrated lens while it is submerged in saline. The hydrogel has a refractive index too similar to that of saline to give a reliable reflex with the lensometer. Furthermore, the base curve cannot be verified by the projection method, because of the small diameter of the lenses. The edge 
Table 1 Preoperative data for 14 primate eyes undergoing intracorneal lens implantation

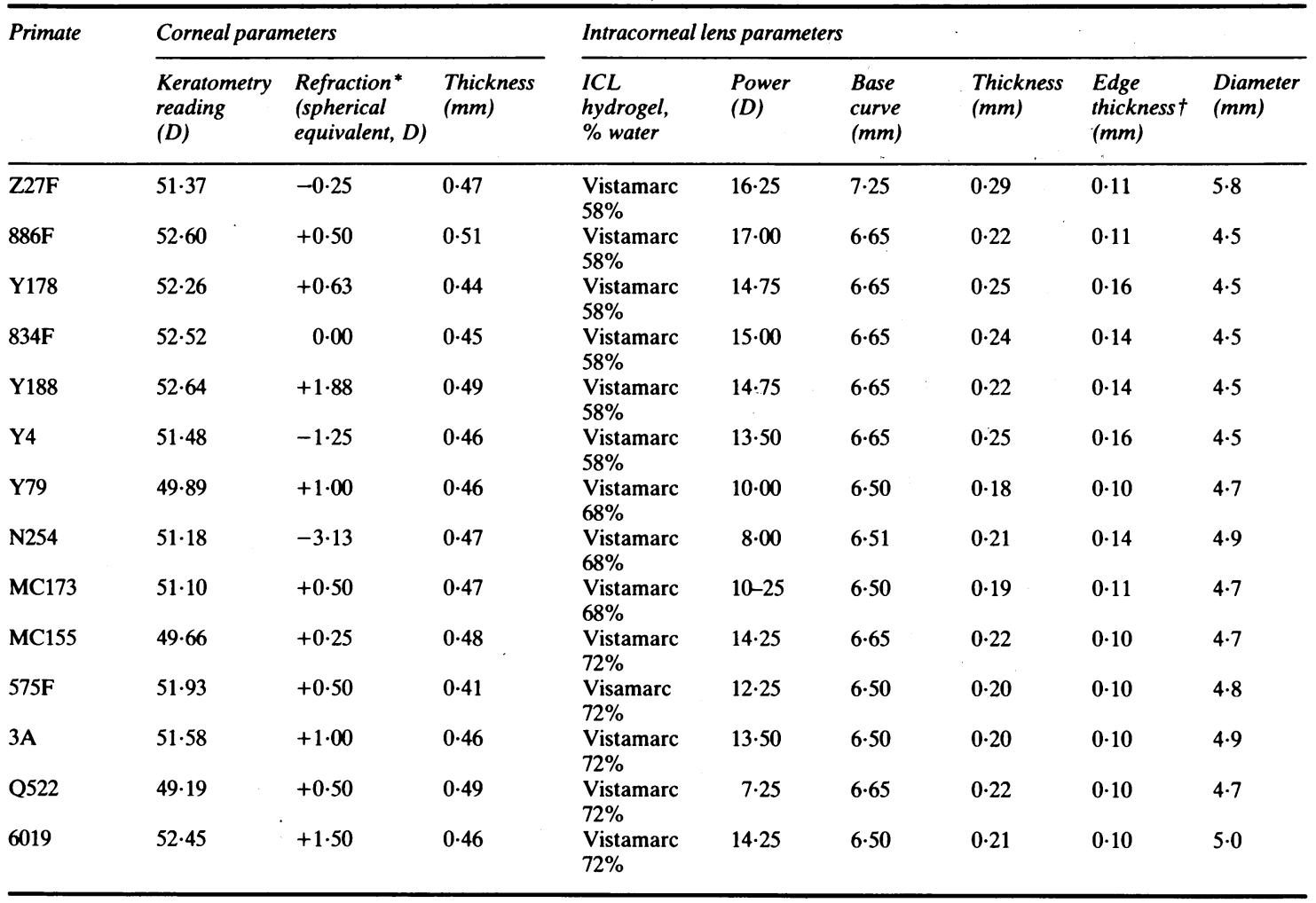

* Expressed as spectacle correction. † Calculated by an algorithm.

thickness was predicted by means of an algorithm described by Watsky et al..$^{5}$ The laboratory specular microscope was used to measure the thickness of the implants.

\section{SURGICAL PROCEDURE}

Fourteen hydrogel intracorneal lenses (ICLs) were implanted in the corneal stroma of 14 adult rhesus monkeys (Macaca mulatta) of both sexes (Table 1). The animals were anaesthetised with intramuscular ketamine $\mathrm{HC} 1(10 \mathrm{mg} / \mathrm{kg})$ with supplements of $5 \mathrm{mg} / \mathrm{kg}$ intravenously. After facial cleaning with providone-iodine solution the animal was placed in a supine position with the head in a sterotactic clamp and an adhesive plastic drape over the face. The plastic drape was cut open over the eye to be operated upon with the opening large enough to minimise interference with the movement of the microkeratome head. A $360^{\circ}$ peritomy exposed the bare sclera so that the suction ring would adhere firmly. A lateral canthotomy with total mobilisation of the lateral canthal ligament was necessary to allow placement of the microkeratome on the globe. A needle-scratch reference mark was made at 120 'clock in the corneal epithelium to allow accurate realignment of the corneal lamellar disc. The eye was proptosed by a retrobulbar injection of $2 \mathrm{ml}$ of balanced salt solution to give access for the microkeratome.

The 10-9 paediatric suction ring of the Barraquer microkeratome (Steinway Instruments, San Diego) was placed on the bare sclera with the conjunctiva retracted. The desired $40 \mathrm{mmHg}$ intraocular pressure was confirmed with the microkeratome applanation unit. The diameter of the lamellar disc to be removed was checked with the $8.5 \mathrm{~mm}$ calibrated sizing applanator. The thickness of the lamellar disc was theoretically set to be $0.35 \mathrm{~mm}$ with a baseplate no. 35 inserted in the microkeratome head along with a new blade for each eye. The microkeratome cut was made with a smooth sweeping movement. The suction was released immediately after the cut and never exceeded 90 seconds in duration. The lamellar disc of the cornea was replaced in its original position guided by the epithelial reference mark at the 12 o'clock position. The area was carefully irrigated, 
and three cardinal 10-0 nylon sutures were placed and tied at the nasal, 12 , and 6 o'clock positions. The temporal suture was put in place and the intracorneal lens inserted from the temporal side after repeat irrigation of the lamellar bed. The ICL was carefully centred and the fourth cardinal suture tied. Sixteen interrupted 10-0 nylon sutures were used in preference to a running suture, so that selected sutures could be removed to reduce neovascularisation or irritation caused by a loose, disrupted suture. After all the sutures were tied, corneal curvature was measured with a Schmirmaul quantitative keratometer mounted on the operating microscope. High astigmatism was corrected by replacing or adjusting the tension of one or two tight sutures. All knots were buried on the peripheral side of the wound. The ICLs central location within the lamellar bed was secured by one 10-0 nylon suture through the anterior stroma and the margin of the ICL at the 12 o'clock position. In practice it was noted that the monkey could dislocate the unsecured ICL during the early postoperative period by rubbing its eye.

The lateral canthotomy was closed with one suture and the conjunctiva was repositioned on to the limbus. Atropine $1 \%$ solution and polymyxin Bbacitracin-neomycin ointment were instilled at the end of the procedure. $1 \mathrm{ml}$ of saline was injected subcutaneously in the upper eyelid to cover the globe and protected it from rubbing during the immediate postoperative period. The monkeys were treated with intramuscular injections of the non-steroidal anti-inflammatory drug flurixin meglumine (Benamine), $1 \mathrm{mg} / \mathrm{kg}$ twice daily for three to five days.

PRE- AND POSTOPERATIVE EXAMINATIONS

Pre-examination anaesthesia and cycloplegia were

Table 2 Postoperative pachymetry* of primate eyes with hydrogel intracorneal lenses

\begin{tabular}{llll}
\hline Primate & $\begin{array}{l}\text { Anterior plus } \\
\text { posterior } \\
(\mathrm{mm})\end{array}$ & $\begin{array}{l}\text { Anterior } \\
\text { lamellae } \\
(\mathrm{mm})\end{array}$ & $\begin{array}{l}\text { Depth of } \\
\text { ICL in } \\
\text { cornea }(\%) \dagger\end{array}$ \\
\hline Y178 & 0.50 & 0.25 & 50 \\
834F & 0.52 & 0.24 & 46 \\
Y188 & 0.57 & 0.36 & 63 \\
Y4 & 0.55 & 0.42 & 76 \\
Y79 & 0.53 & 0.31 & 58 \\
N254 & 0.56 & 0.30 & 54 \\
MC173 & 0.56 & 0.30 & 54 \\
MC155 & 0.57 & 0.39 & 68 \\
575F & 0.53 & 0.31 & 58 \\
3A & 0.53 & 0.36 & 68 \\
Q522 & 0.55 & 0.31 & 56 \\
\hline
\end{tabular}

* Average of last three examinations.

$\dagger$ Anterior lamella thickness divided by anterior plus posterior lamella thickness. performed as described by McCarey et al. ${ }^{2}$ The examination included slit-lamp microscopy, central pachymetry with the Haag-Streit I unit, central keratometry, retinoscopy, and slit-lamp photography. All monkeys had at least one preoperative examination. Postoperative examinations were performed on the 3rd and 7th, 14th and 21st days after surgery, monthly for six months, and then bimonthly. During the immediate postoperative period the animals had a partial examination (that is, biomicroscopy and, if possible, estimated pachymetry and keratometry) on the 3rd and 7th postoperative day as well as removal of loose sutures. The suture through the ICL was removed on the 7th postoperative day. After three weeks all remaining sutures were removed. The retinoscopy data reported in this manuscript (except for Figs. 2 and 3) are the average value from the last three examinations and are expressed as the spectacle correction. The theoretical chance in refraction was calculated by means of an algorithm developed by Watsky et al. ${ }^{5}$

\section{Results}

\section{POPULATION STUDIED}

The preoperative corneal measurements of the 14 corneas (Table 1) showed central keratometry readings averaged $51 \cdot 40 \pm 1 \cdot 12 \mathrm{D}( \pm \mathrm{SD})$ or radius $6.57 \pm 0.15 \mathrm{~mm}$, spherical equivalent refractions averaged $0 \cdot 26 \pm 1 \cdot 23 \mathrm{D}$, and central corneal thickness averaged $0.46 \pm 0.02 \mathrm{~mm}$.

Three monkeys were omitted from the postoperative refractive results. Animal Z27 developed a corneal ulcer and the ICL was removed at week 12 . One animal, $886 \mathrm{~F}$, died of aspiration pneumonia at week 18 . Severe corneal vascularisation developed in response to fierce eye rubbing by animal 6019 , and the ICL was removed at week 24 . The results of the remaining 11 animals are presented with follow-ups ranging from 49 to 53 weeks.

\section{PACHYMETRY}

The preoperative full corneal thickness for the 11 eyes averaged $0.46 \pm 0.02 \mathrm{~mm}( \pm \mathrm{SD})$. After approximately one year of postoperative follow-up the same set of eyes minus the implant thicknesses averaged $0.54 \pm 0.02 \mathrm{~mm}$. Pachymetry showed increasing thickness postoperatively in both operated and contralateral unoperated eyes. In five out of 10 animals the experimental cornea was signficantly $(t$ test, $\mathrm{p}<0.005)$ thicker than the control cornea. No animals showed a significant decrease of total stromal thickness following implantation of a hydrogel lens. Since the implant separated the anterior and posterior lamellae, the postoperative values include two subjective pachymetry endpoint decisions. The thickness of the 
Table 3 Postoperative data for 11 primate eyes with hydrogel intracorneal implants

\begin{tabular}{|c|c|c|c|c|c|c|c|c|}
\hline \multirow[t]{2}{*}{ Primate } & \multirow{2}{*}{$\begin{array}{l}\text { Week } \\
\text { postoperative }\end{array}$} & \multicolumn{3}{|c|}{ Refractive change* } & \multicolumn{4}{|c|}{ Keratometry } \\
\hline & & $\begin{array}{l}\text { ICL power } \\
\text { (D) }\end{array}$ & $\begin{array}{l}\text { Theoretical } \\
\text { (D) }\end{array}$ & $\begin{array}{l}\text { Measured } \dagger \\
\text { (D) }\end{array}$ & $\begin{array}{l}\text { Yield } \\
(\%)\end{array}$ & $\begin{array}{l}\text { Theoretical } \\
\text { (D) }\end{array}$ & $\begin{array}{l}\text { Measured }{ }^{*} \\
\text { (D) }\end{array}$ & $\begin{array}{l}\text { Yield } \\
(\%)\end{array}$ \\
\hline Y178 & 52 & $14 \cdot 75$ & $12 \cdot 20$ & $13 \cdot 13 \pm 1 \cdot 34$ & $108 \pm 11$ & $10 \cdot 57$ & $9 \cdot 07 \pm 1 \cdot 50$ & $86 \pm 15$ \\
\hline $834 \mathrm{~F}$ & 51 & $12 \cdot 50$ & $12 \cdot 31$ & $12.49 \pm 1.67$ & $101 \pm 14$ & $10 \cdot 76$ & $6 \cdot 94 \pm 3 \cdot 16$ & $64 \pm 29$ \\
\hline Y188 & 52 & $10 \cdot 50$ & $11 \cdot 94$ & $10 \cdot 63 \pm 0 \cdot 42$ & $89 \pm 4$ & $10 \cdot 11$ & $6 \cdot 83 \pm 0 \cdot 32$ & $67 \pm 4$ \\
\hline Y4 & 48 & $13 \cdot 50$ & $10 \cdot 94$ & $9 \cdot 18 \pm 0 \cdot 55$ & $86 \pm 6$ & 9.09 & $4 \cdot 35 \pm 0 \cdot 39$ & $48 \pm 4$ \\
\hline Y79 & 52 & $10 \cdot 00$ & $7 \cdot 79$ & $7 \cdot 85 \pm 1 \cdot 71$ & $100 \pm 22$ & $7 \cdot 76$ & $6 \cdot 85 \pm 0 \cdot 45$ & $92 \pm 6$ \\
\hline N254 & 52 & $8 \cdot 00$ & $6 \cdot 40$ & $8 \cdot 32 \pm 0.44$ & $125 \pm 14$ & $6 \cdot 22$ & $8.19 \pm 0.97$ & $131 \pm 15$ \\
\hline MC173 & 53 & $10 \cdot 25$ & $7 \cdot 88$ & $9.93 \pm 1.02$ & $127 \pm 13$ & $7 \cdot 57$ & $9 \cdot 41 \pm 1 \cdot 66$ & $124 \pm 22$ \\
\hline MC155 & 53 & $14 \cdot 25$ & 5.91 & $9.06 \pm 0.95$ & $153 \pm 16$ & $5 \cdot 78$ & $6 \cdot 58 \pm 0 \cdot 35$ & $114 \pm 5$ \\
\hline $575 \mathrm{~F}$ & 49 & $11 \cdot 25$ & $9 \cdot 39$ & $11.37 \pm 0.63$ & $121 \pm 6$ & $9 \cdot 13$ & $6 \cdot 81 \pm 0 \cdot 12$ & $75 \pm 1$ \\
\hline $3 \mathrm{~A}$ & 52 & $13 \cdot 50$ & $10 \cdot 35$ & $9.75 \pm 0.44$ & $94 \pm 4$ & 9.96 & $7 \cdot 83 \pm 0 \cdot 45$ & $79 \pm 5$ \\
\hline Q522 & 51 & $7 \cdot 25$ & $5 \cdot 21$ & $10 \cdot 46 \pm 0 \cdot 23$ & $201 \pm 3$ & $5 \cdot 30$ & $7 \cdot 23 \pm 0.63$ & $136 \pm 10$ \\
\hline
\end{tabular}

${ }^{*}$ Expressed as spectacle correction. $†$ Average of last three examinations.

anterior lamellae excised with the no. 35 microkeratome base plate should be $0.35 \mathrm{~mm}$, but it measured an average of $0.32 \pm 0.06 \mathrm{~mm}$ (mean $\pm \mathrm{SD}$ ) (Table 2). The depth of the implant in the cornea averaged $59 \pm 9 \%$ (mean $\pm \mathrm{SD}$ ) relative to its postoperative thickness (anterior + posterior lamellae).

CHANGES IN CORNEAL POWER AND REFRACTION The postoperative data in Table 3 contain the theoretical and refractive changes as well as the measured change in refraction and keratometry (average of the last three examinations) for 11 animals. The average postoperative follow-up was $51 \pm 2$ weeks with the refractive yield being $118 \pm 34 \%$ and the keratometric yield being $92 \pm 30 \%$. The measured versus theoretical expected refractive changes had a linear regression line correlation coefficient of $0 \cdot 74$, whereas the respective keratometric data had a correlation coefficient of $0 \cdot 04$. The measured refractive changes of each animal was graphed for the 51 week follow-up (Figs. 1 and 2). The measured refraction became stable within 2 to 3 dioptres after 20 weeks. When data from the same animal group were graphed by the refractive yields (Fig. 3), a greater spread was shown. Although many animals had yields between 80 and $120 \%$ after 20 weeks, some of the higher water content lenses resulted in much higher yields (Fig. 4).

\section{SLIT - LAMP MICROSCOPY}

The hydrogel ICLs were tolerated well within the corneas of the animals. Most animals showed a slight grey lining at the implant margin. Hydrogel lenses with an abruptly cut edge instead of a rounded, tapered, or buffed edge tended to have more of this build-up. At the interface of the ICL and the stroma refractile particles could be detected in some animals. At the site of the microkeratome cut scarring to some extent was present in all animals. Epithelial downgrowth into the bed of the microkeratome cut did not occur in any of the animals. Animal Z27 was removed from the study at 12 weeks postoperatively because an ulcer developed at the margin of the implant. The implant was removed and subsequently the cornea became clear. After 24 weeks of observation the

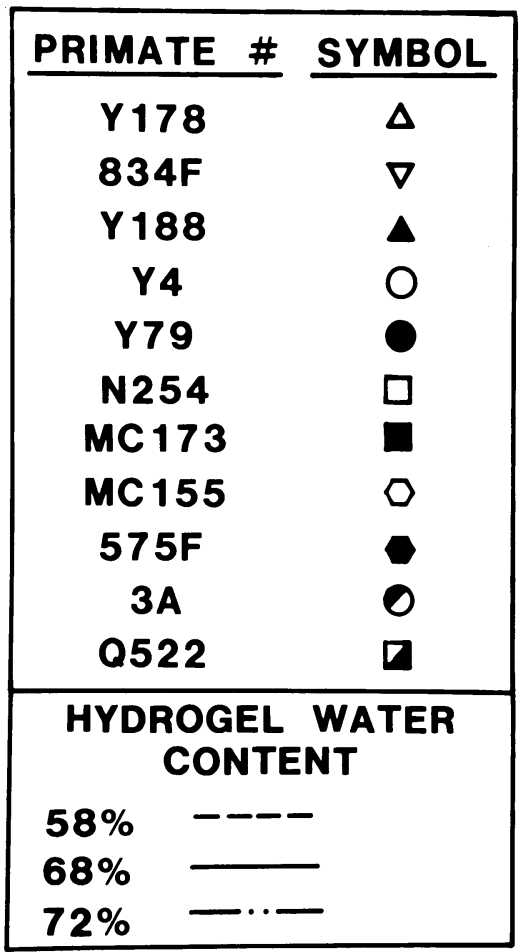

Fig. 1 Key of symbols used in Figs. 2, 3, and 4. 
Fig. 2 Long-term follow-up of the changes in the measured refractions relative to the preoperative refractions illustrates the stability refraction of the corneal/hydrogel implant complex. Symbols as in Fig. 1.

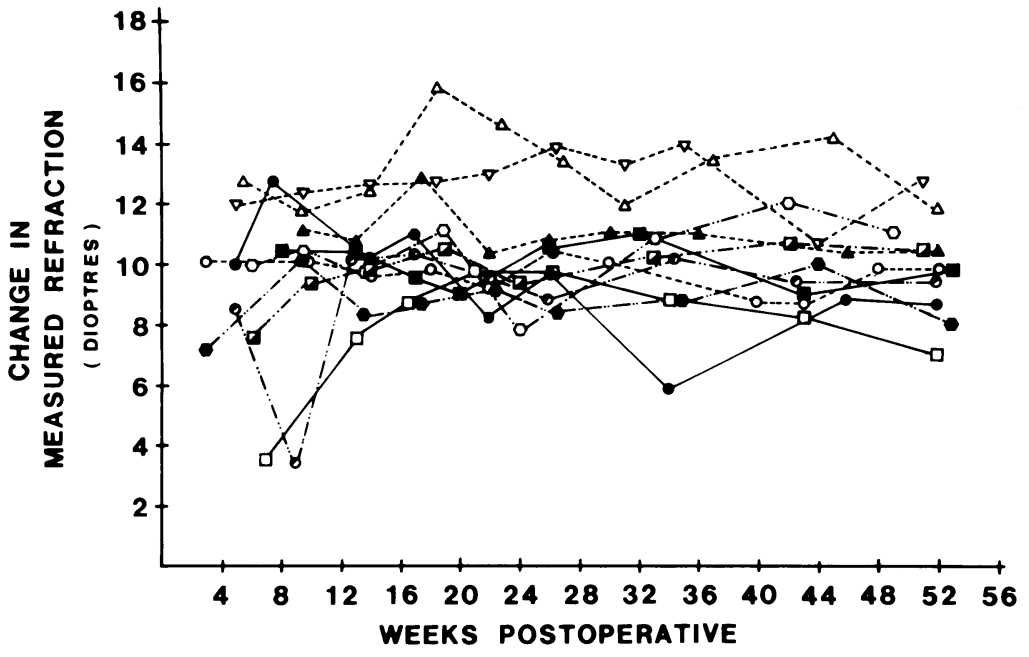

implant was removed from the cornea of animal 6019 . There was marked vascularisation and more than average scarring because this animal was rubbing his eye in an unusually fierce manner during the past surgical period.

\section{Discussion}

Since the monkey eye dimensions and facial structure are different from those in man, we had to adjust the
Barraquer microkeratome technique. We found it necessary to use the paediatric microkeratome ring with a $360^{\circ}$ conjunctival peritomy, a lateral canthotomy, and a retrobulbar injection of saline in order to gain exposure to the cornea and adequately to secure the microkeratome suction ring. Even then a smooth path of the microkeratome was sometimes impeded by orbital and nasal structures. After surgery the monkey's propensity to rub the irritated eye was only somewhat reduced by a saline injection to expand the
Fig. 3 When the long-term data are graphed by the calculated refractive yield (measured refractive change divided by the theoretical change) the data appears to have an unacceptably excessive spread. Symbols as in Fig. 1.

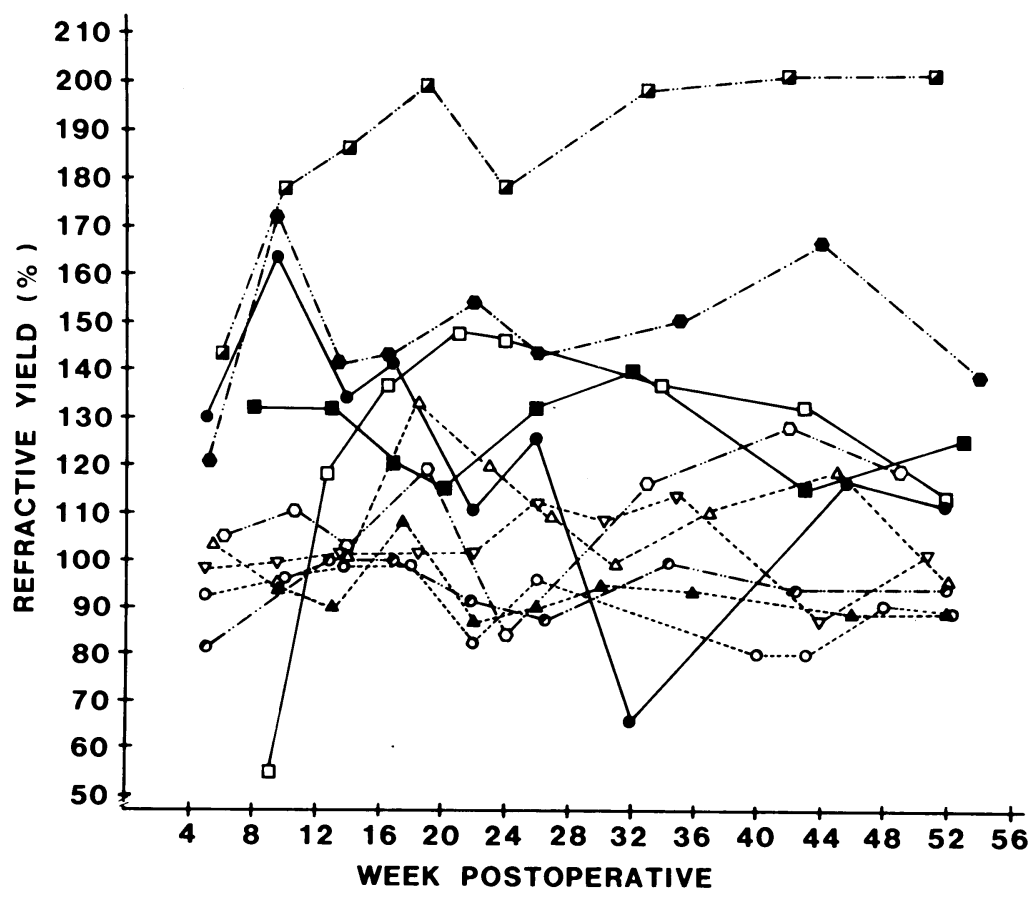




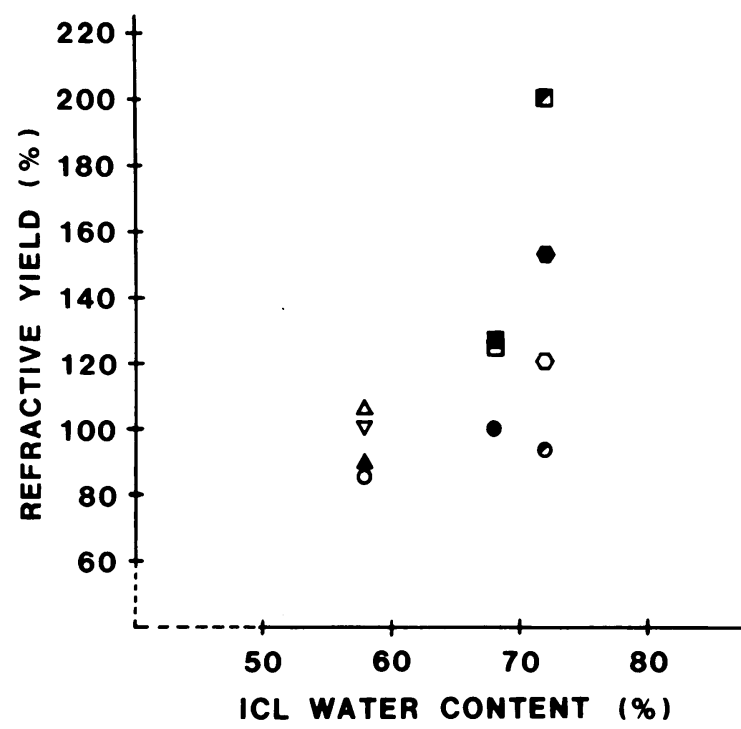

Fig. 4 Summary of the refractive yields (measured refractive change divided by the theoretical change) relative to the water content of the hydrogel implant illustrates the increasing variability with increasing water contents which helps to explain the spread in Fig. 2. Symbols as in Fig. 1.

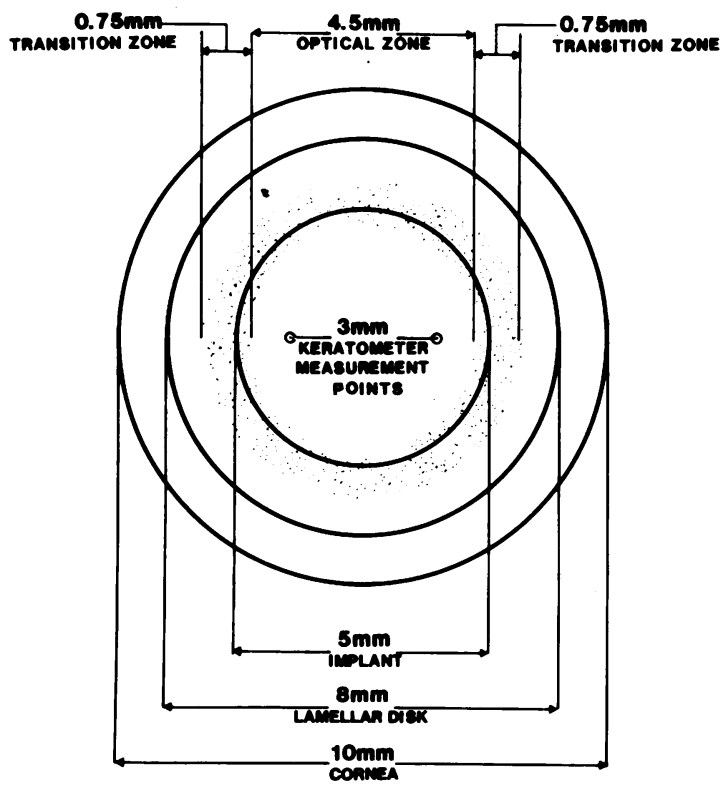

Fig. 5 The corneal curvature measurement by Bausch and Lomb keratometer is taken at two points on a circle with a diameter of $2 \cdot 8$ to $3 \cdot 1 \mathrm{~mm}$. The estimated irregular transition zone at the edge of the ICL is represented as a dotted area. The keratometry readings should be centred over the ICL, which is very difficult to see, since even slight decentration will cause false readings in the transition zone. upper lid over the globe and frequent examinations to remove the irritating loose sutures.

The keratometry measurements are difficult on monkeys because of the optical principles of the instrument in combination with the animal model. The Bausch and Lomb keratometer with the addition of a $+1 \cdot 25 \mathrm{D}$ auxiliary lens to extend the instrument's dioptric range measures the corneal curvature from points on a 2.8 to $3.1 \mathrm{~mm}$ circle (the range depending on the radius of curvature), (Fig. 5). The anaesthetised monkey cannot fixate on the central light of the keratometer, and therefore the exact surface location being measured is unknown. The ICLs have diameters of approximately $5 \mathrm{~mm}$, with the optical zone being approximately $4.5 \mathrm{~mm}$. If the keratometry is not precisely centred over the implant, which cannot be visualised through the keratometer, there will be a large variation of the readings. This is illustrated by the poor correlation between expected and measured keratometry values. Keratoscope photographs have the same problem of centration.

Hydrogel ICL implantation in lamellar pockets in primate eyes does not change the corneal surface curvature $^{2}$ as it does in rabbits. ${ }^{1}$ A microkeratome dissection is necessary to allow the hydrogel implant to be placed between smooth surfaces in the corneal stroma at a level of even depth. Dissecting the anterior stromal lamella free from the cornea and placing it over the hydrogel lenticule results in the outer surface conforming to a predictable curvature. When a lenticule (positive or negative power) is introduced in the stroma to change the anterior corneal curvature, the arc length on the corneal surface will increase. The anterior stromal layers, including Bowman's layers of the primate eye, are not elastic as in the rabbit eye. These layers will not stretch to a larger arc length over an implanted wedge.

The relationship between the water content of the implanted lenticules and the yield of refractive change was examined (Fig. 4). The 58\% water content lenticules have the best predictability (yields close to $100 \%$ ). The $68 \%$ and $72 \%$ water content lenticules have less ideal yields. We think these differences are due to: (1) errors in the calculation of the expansion factor, and (2) variation of hydration of the materials by the manufacturer. Neither the hydration of each lot of lenses nor the refractive power was double checked in this study prior to implantation, for reasons explained above and in an earlier paper, ${ }^{2}$ Another cause for differences in results between materials with various water contents might be a change in dimension, that is, hydration, of the hydrogel material within the corneal stroma due to mechanical compression or stromal swelling prassure. These factors have been considered in a sep- 
arate study. ${ }^{6}$ The conclusion of that study was that hydrogel materials up to a water content of $70 \%$ can be considered stable within the corneal stroma. Materials of higher water content undergo a hydration decrease by a few percent when exposed to physiological swelling pressures. This observation does not, however, explain the higher yields found in this study for the higher water content Vistamarc materials.

Pachymetric measurements have shown that the anterior lamellae are thinner than the intended 0.35 $\mathrm{mm}$ that would be expected with the microkeratome fitted with the no. 35 base plate (Table 2). This may be explained by the earlier observations that the hydration of the stroma anterior to the ICL decreases. ${ }^{\prime}$

In analysis of our pachymetric data we have considered mainly the difference between the stromal thickness of the experimental eye and the control eye at each measuring point. Both the experimental corneal thickness and the control values increased significantly in thickness over time. Subtracting the two values at each time point illustrated that half the experimental group was significantly greater than the control eye and the other half was statistically equal to the control. The increase in the control thickness over time can be ascribed to either age changes in the animal or measurement error, of which the latter is probably the most important factor. The retinoscopic reflex did not deteriorate during the follow-up period.
In conclusion, the implanted hydrogel materials were well tolerated in the primate corneal stroma; 11 out of 14 implants have been technically successful. The refractive change was stable after ICL implantation. The measured refractive change correlated well with the calculated refractive change in most animals, but the higher water content hydrogel implants gave less predictable results. Keratometry was an unreliable measure of the change in corneal power in this experimental primate study.

This work was supported by NIH Grant no. EYO3696 and by a grant from IOLAB Corp.

\section{References}

1 McCarey BE, Andrews DM. Refractive keratoplasty with intrastromal hydrogel lenticular implants. Invest Ophthalmol Vis Sci 1981; 21: 107-15.

2 McCarey BE, van Rij G, Beekhuis WH, Waring GO. Hydrogel keratophakia via a freehand pocket dissection in the monkey model. BrJ Ophthalmol 1986; 70: 187-91.

3 Barraquer JT. Symposium on medical and surgical diseases of the cornea. St Louis: Mosby, 1980: 450-79.

4 Binder PS, Zwada EY, Deg JK, Baumgartner SD. Hydrophylic lenses for refractive keratoplasty: the use of factory lathed materials. Contact Lens Association of Ophthalmologists Journal 1984; 10: 105-11.

5 Watsky M, McCarey BE, Beekhuis WH. Hydrogel keratophakia computing optical modifications. Invest Ophthalmol Vis Sci 1985; 26: 240-3.

6 Beekhuis WH, McCarey BE, Baum J. Hydration stability of intracorneal hydrogel implants. Invest Ophthalmol Vis Sci in press.

Accepted for publication 2 July 1985. 